\title{
Social Learning Class Topper Optimization (SL-CTO) Based Hop Localization Technique for Wireless Sensor Network
}

\section{TAPAN KUMAR MOHANTA}

ICFAI University Tripura

Dushmanta Kumar Das ( $\square$ dushmantakumardas29@gmail.com )

National Institute of Technology Nagaland https://orcid.org/0000-0001-8030-6242

\section{Research Article}

Keywords: Dumb nodes, Beacon nodes, SL-CTO Algorithm, DV-Hop Algorithm

Posted Date: August 9th, 2021

DOl: https://doi.org/10.21203/rs.3.rs-659985/v1

License: (c) (1) This work is licensed under a Creative Commons Attribution 4.0 International License.

Read Full License 


\title{
Social Learning Class Topper Optimization (SL-CTO) based hop localization technique for Wireless Sensor Network
}

\author{
Tapan Kumar Mohanta . \\ Dushmanta Kumar Das
}

\begin{abstract}
To address the current situation limitation of traditional DV-Hop, we suggested a DV-Hop localization based on a rectification factor using the Social Learning Class Topper Optimization (SL - CTO) algorithm in that paper. In order to adjust the number of hops between beacon nodes, we have implemented a rectification factor in the suggested method. By measuring the dimensions of all the beacons at dumb nodes, the suggested algorithm decreases communication among unknown or dumb and beacon nodes. The model of network imbalance, It is often considered to be demonstrate a applicability of the Proposed approach in the anisotropic network. Simulations have been performed on LabVIEW@2015, and Comparisons were made with conventional DV-Hop, particle swarm optimization-based DV-Hop and runnerroot optimization-based DV-Hop for our proposed algorithm. In comparison to current localization methods, simulation outcomes showed that the proposed localization technique reduces computing time, localization error variance and localization error.
\end{abstract}

Keywords Dumb nodes · Beacon nodes · SL-CTO Algorithm · DV-Hop Algorithm

\section{Introduction}

Low-priced, low Consumption of power and nodes for self-reconfigurable compose the sensor network. These nodes of the sensor detect a destination of the event and forward the data sensed to the main location referred to as the sink node [1]. Easy distribution and Low-sensor node costs make WSN dignified of applications such as army affairs, environmental issues, health services,

Department of ECE, ICFAI University

Kamalghat, Tripura, 799210, India, e-mail: tapank838@gmail.com

Department of Electrical and Electronics Engineering, National Institute of Technology

Nagaland, 797103, India. e-mail: dushmantakumardas29@gmail.com.

Received: date / Accepted: date 
traffic calming, national security motive, etc. Physical location for certain applications of the relevant events $[2,3]$. Event data has no significance without its location information. One of the easiest methods for node localization is manual deployment of sensor nodes, but it is not feasible in remote regions for large-scale deployment. The simplest system for localization is the global positioning system (GPS), but the cost may boost of the network and even consuming more energy [4]. One of most cost-effective ways to locate sensor nodes is to activate some GPS nodes, also known as beacon nodes. Manually deploying sensor nodes and recording their coordinates is another method of localization. However, the network has a significant number of nodes, this technique is not universal, and human participation in some monitoring regions is not practical. Via GPS or manual deployment, the widely used node localization scheme is to obtain the coordinates of some nodes (called beacon nodes or anchor nodes) and then use localization algorithms to measure the coordinates of other nodes (called unknown nodes or dumb nodes). Such anchor nodes understand their precise location and tries to retain their location for the unknown nodes [5,6]. Many localization strategies have been suggested for WSNs [7] to solve these position estimation issues. These methods of localization are classified as dependent on range and free of range. The range-based method uses an algorithm based on constraint measurements and typically requires higher distance measurement costs [8]. The range-based approach is split into few types: Time Of Arrival (TOA) [9], Time Difference Of Arrival (TDOA) [10], Received Signal Strength Indicator (RSSI) [11], and Arrival Of Angle (AOA) [12,13]. These techniques demonstrate good accuracy of localization, but require additional hardware to estimate of nodes. Range-free destination technology, on the other hand uses the algorithm of approximate distance as well as hop number between beacon and dumb nodes to approximate the location of the locate nodes. This method lowers the cost because no additional hardware is required [14]. One of the commonly used range-free localization algorithms [15] is the DV-Hop algorithm. This algorithm, however, faces low precision of localization $[16,17]$.

Nature has made a huge contribution to success of the modern algorithms for optimization and has also offered a solution to several complex engineering problems. There are numerous algorithms inspired by nature, just like the Genetic Algorithm (GA), Artificial Bee Colony (ABC), Particle Swarm Optimization (PSO), Simulated Annealing (SA) and Ant Colony Optimization (ACO). It has been found that the PSO is algorithm of quick processing. However, it is difficult for the algorithm to escape, when it is stuck in the local minimum. SA has random initial disadvantages and there's fewer chances of finding worldwide solutions. It is also observed that Genetic Algorithm (GA) has advantages over PSO. The approach is within an user defined boundary area. However, Genetic Algorithm (GA) has disadvantages of being sluggish and has better scope. One of those kinds of algorithm, inspired by strawberry plants, is also the Runner-root algorithm (RRA), used to solve multivariable problems. Half of the agents that become vulnerable are discarded in this 
algorithm and good agents are replicated at any iteration. From the above discussion, It is observed that the usefulness of these strategies depends also on problem under consideration. Social learning plays a significant role among social animals in the learning of actions. Social learning has the benefit of encouraging people to learn habits from the others without deducting the cost in specific trials-and-errors, as opposed to individual (asocial) learning. In order to build a social learning CTO, this paper integrates social learning process into class topper optimization (CTO). Each student in the proposed SL-CTO learns from good students, unlike CTO variants where students are modified based on knowledge, including the best student learned by the entire class (class topper) and the best student learned by each section (section topper). Furthermore, the proposed SL-CTO implements a dimension-dependent method of parameter control to ease the burden of parameter settings. We have been proposing a SL-CTO-based enhanced DV-Hop algorithm in WSNs to resolve the disadvantages of conventional DV-Hop localization. This paper's major contributions are as follows:

1. By calculation of the hop size with all beacons at dumb nodes, the proposed algorithm will reduce the amount of messages sent among dumb or unknown nodes and beacon nodes. This saves time of localization and localization resources, which also minimizes the proposed algorithm's Communication systems costs.

2. We also included a rectification factor in the suggested technique to modify hop dimensions of a beacon nodes.

3. To model the impact of the anisotropic environment, the imbalance variable (degree of irregularity) has also been integrated.

4. To correct the approximate locations of the dumb nodes, the SL-CTO optimization algorithm has been used to reduce localization error.

The results of the simulation indicate that, the current range-free localization algorithm, Our suggested algorithm for localization reduces position error, variance in localization error and time required for computation.

The remaining paper is organized as follows: Section 2 includes related research. The typical position of DV-Hop is addressed in Section 3. The SLCTO-based DV-Hop localization proposed is listed in Section 4. The radio irregularity effect is mentioned in Section 5. Section 6, explains the output metrics. Results from the simulation are shown in Section 7. Finally, in Section 8 , conclusions are drawn.

\section{Related works}

Numerous optimization-based algorithms have been developed to solve localization issues.

Throughout this article, we had also done a comprehensive survey of the studies' work in the position of optimization based on hop localization. It primarily 
covers the tasks for which the CTO method is employed in hop-based localization as well as the DV-Hop algorithm changes. A amount with DV-Hop-based algorithms for range-free method were studied in this section. In order to estimate the position of the nodes, Chen and Zhang [18] distributed a few other anchor nodes only at boundary of a monitoring areas. Finally, to reduce the localization error for the algorithm, Particle Swarm Optimization (PSO) is used. For isolated areas, the deployment of anchor nodes at the boundary of the sensor network in this paper is not sufficient.

To minimize the localization error, Kumar et al. [1] introduced a energyefficient range-free technique. Here, by asking unknown nodes about their coordinates, anchor nodes broadcast their location to dumb nodes. The hop size for a beacon nodes are defined on the dumb nodes. This method efficient builds the algorithm's energy efficient.

Zaidi et al. [19] suggested a new algorithm in which dumb nodes would use locally available information to locate their positions, thus eliminating some redundant depreciation and energy costs sustained if it was necessary to share data between nodes. This paper does not demonstrate the impact of network irregularities on the localization algorithm. An advanced range-free method using genetic algorithms was proposed by Sharma and Kumar [20].

They change the average hop size of the anchor nodes by optimizing the correction factor, and the modified hop length is configured further by a line search algorithm. The efficiency of localization has been increased by using a genetic algorithm. However, because of gaps, non-uniform node distribution network randomness, and abrupt radio patterns, the suggested algorithm efficiency can be degraded. To use the Grey Wolf algorithm to get a more accurate estimate of the average hop distance as determined by each beacon node, Kaur et al. [21] suggested of grey wolf optimization. The algorithm has been shown to provide greater precision with a small increase in the cost of computing. Using PSO, an improved DV-Hop localisation algorithm was proposed by Singh and Sharma [22]. They have measured the value of hop count and minimum hop size in the proposed algorithm, measuring the location and performing error evaluation. The method developed utilizes the hop size of the anchor from which the dumb node defines its reach. In addition, with the PSO strategy, the positions of unknown nodes are enhanced.

Kanwar and Kumar [6] have suggested a range-free localization using runnerroot algorithms. They change the average hop size of the anchor nodes by refining a correction factor, and the changed hop size is further optimised by a line search algorithm. The precision of localization is further improved utilizing a runner-root method. However, it is possible to degrade the efficiency of the proposed algorithm as a result of holes, abnormal radio patterns, and non-uniform node delivery network sparsity.

We inferred from the above reviews that range-free localization algorithms still need improvements in localization accuracy terms. This motivates us to propose a new algorithm using SL-CTO for DV-Hop localization. 


\section{Algorithm of conventional DV-Hop}

The localization technique is essentially a free localization technique that is based on a protocol for distance routing. For the number of hops of beacon nodes as well as the minimum hop distance besides WSNs, calculating the distance between dumb nodes or unknown nodes and beacon nodes is used. Different paths form in a network topology among dumb nodes and beacon nodes that are not linear due to non-uniform connectivity with wireless sensor nodes. Therefore, Some errors have been identified at the period of the algorithm in the node position method [6].

Step 1 The minimum hop amount is defined for unknown nodes and beacon nodes of step 1. By transmitting signals through beacon nodes by vector protocol system, the neighbouring nodes can be shown their location,Information exists in the form of $\mathrm{Hi}, a i, b i, i d$, in which $i d$ will be the identity, ai, bi have been coordinates, and $H i$ has been the hop count for the $i$ beacon node.

First, 0 is set of the value for $H_{i}$ [23]. The nodes obtain data from the broadcast and record the hop amount and localization of the vector's beacon nodes. The value of $H_{i}$ must be increased by 1 through this process [20]. In this update process, if any node receives the same $i d$ group, the new received data will be compared with the original value of $H_{i}$. The nodes obtain broadcast data and keep track of the hop amount and localization for the beacon nodes of the vector.

Step 2 Minimum hop count and average hop distance were determined to find the distance between unknown nodes and beacon nodes. In this method, the average hop distance for the entire network can be determined by obtaining the position and hop amount for nodes of beacons, as described in the previous stage. Then this data is transmitted to the entire network. For most nodes, The minimum hop distance data from the a beacon node closer to them is also necessary to obtain [24]. The following equation provides the typical distance of the hop $\left(j p_{i}\right)$ and the hop range $\left(p_{i}\right)$ between the $i\left(a_{i}, b_{i}\right)$ beacon node and the other (beacon) node $j\left(a_{j}, b_{j}\right)$ can just be computed as:

$$
j p_{i}=\frac{\sum \sqrt{\left(a_{i}-a_{j}\right)^{2}+\left(b_{i}-b_{j}\right)^{2}}}{\sum h(i j)} .
$$

The distance between beacon nodes and dumb nodes is expressed in the following formula.

$$
p_{i u}=j p_{i u} \times H o p_{\text {min }}
$$

where $j p_{i}$ is the average hop distance, $H o p_{\text {min }}$ is the hop count between the $i$ beacon nodes and the $u$ dumb nodes. 
Step 3 Let the $U$ dumb node coordinates be $(a, b)$, and the $i^{\text {th }}$ beacon node coordinates be $\left(a_{i}, b_{i}\right)(1 \leq i \leq n)$ analyzed in stage 3 . Correspondingly, the distance between the beacon node $i^{\text {th }}$ to the unknown or dumb node $U$ is $p_{i}(1<i<n)$. The coordinates of the dumb node have been determined as follows:

$$
\begin{aligned}
& \left(a-a_{1}\right)^{2}+\left(b-b_{1}\right)^{2}=p_{1}^{2} ; \\
& \left(a-a_{2}\right)^{2}+\left(b-b_{2}\right)^{2}=p_{2}^{2} \text {; } \\
& \left(a-a_{n}\right)^{2}+\left(b-b_{n}\right)^{2}=p_{n}^{2} ; \\
& a_{1}^{2}-a_{n}^{2}+2\left(a_{1}-a_{n}\right) a+b_{1}^{2}-b_{n}^{2}-2\left(b_{1}-b_{n}\right) b \\
& =p_{1}^{2}-p_{n}^{2} \text {; } \\
& a_{n-1}^{2}-a_{n}^{2}+2\left(a_{n-1}-a_{n}\right) a+b_{n-1}^{2}-b_{n}^{2}-2\left(b_{n-1}\right. \\
& \left.-b_{n}\right) b=p_{n-1}^{2}-p_{n}^{2} \text {; }
\end{aligned}
$$

(3) can be arranged from $S A=T$ in the matrix, where

$$
\begin{aligned}
S & =\left[\begin{array}{cc}
2\left(a_{1}-a_{n}\right) & 2\left(b_{1}-b_{n}\right) \\
2\left(a_{2}-a_{n}\right) & 2\left(b_{1}-b_{n}\right) \\
\ldots \ldots \ldots \ldots \ldots \ldots \ldots \ldots \ldots \ldots \\
2\left(a_{n-1}-a_{n}\right) & 2\left(b_{1}-b_{n}\right)
\end{array}\right], \\
T & =\left[\begin{array}{c}
a_{1}^{2}-a_{n}^{2}+b_{1}^{2}-b_{n}^{2}+p_{n}^{2}-p_{1}^{2} \\
a_{2}^{2}-a_{n}^{2}+b_{2}^{2}-b_{n}^{2}+p_{n}^{2}-p_{2}^{2} \\
\ldots \ldots \ldots \ldots \ldots \ldots \ldots \ldots \ldots \ldots \ldots \ldots \ldots \\
a_{n-1}^{2}-a_{n}^{2}-b_{n-1}^{2}-b_{n}^{2}+p_{n}^{2}-p_{n-1}^{2}
\end{array}\right], \\
A & =\left[\begin{array}{c}
a \\
b
\end{array}\right],
\end{aligned}
$$

Finally, the last square approximation is used to calculate the unknown node coordinates as given below:

$$
A=\left(S^{T} S\right)^{-1} S^{T} T
$$

\section{A Social Learning Class Topper Optimization (SL - CTO) Algorithm}

Below will be a short summary for the sociological context of the proposed SL - CTO. A detailed explanation of SL - CTO will then be provided in conjunction with a study of complexity and integration in computation. Several 
British birds have been seen opening milk bottles for 1921 in the little town for Swaythling. Such findings were continuously reported in 25 years from numerous other locations in the United Kingdom and even in some other region of the continent of Europe. It's the first proof of learning socially [25], in which it is assumed that birds learn to open bottles of milk through observations and experiences for other birds, rather than learning on their own [26]. Different mechanisms in social learning theory have been suggested and debated over the past decades, such as incentive development and local improvement [27], observational conditioning [28], contagion [29] and social facilitation [30]. Imitation, which is considered distinctive from other mechanisms of social learning, is among these mechanisms [31], is the most interesting mechanism of social learning, since imitation, which occurs across a whole society, may lead to behavioural similarities at the population level, such as community or tradition [32]. Such parallels in population-level can mean the integration of a complex system, hence having the evolutionary algorithm with its critical applicability. In [33], the authors addressed in depth the various meanings for imitation, of the definition for Mitchell is known to be really applicable to animals and devices [34], in which imitation is assumed to be a method of producing a similar copy of the model. In [32], imitation is defined as an imitator procedure that copies part of a demonstrator's behaviour through observation. To replace the updating rules in Class Topper Optimization (CTO), We propose few other new methods for learning that are influenced by social learning.

Like the CTO, the proposed SL - CTO initialises a student with a section where the size of the student and the performance index (PI). It holds a randomly initialised learning behaviour for each section, which represents a student solution to the optimization issue. Each student will be allocated with knowledge enhancement value calculated from learning form as reward input from the environment. The student is then sorted according to the learning values of a growing order of students. Each student can then correct their behaviors by learning from those students (demonstrators i.e ST and CT).

The flowchart of SL - CTO is shown in Fig. 1. An simple explanation of the mechanism of learning. First, graded according to every student makes an effort to gain understanding that leads to improvement in class topper results. Then each students, except for the class topper, will benefit greatly from the best students. Enhancement of knowledge at section level and at student level, two types of knowledge changes are presented. The student's efficiency is enhanced by learning from the best student of that class [35].

An imitator's social learning system will learn the actions of various demonstrators [36] in the following manner:

$$
L_{i, j}{ }^{T+1}=\left\{\begin{array}{cc}
L_{i, j}^{T}+\Delta L_{i, j}^{T+1}, & \text { if } \mathrm{p}_{i}(t) \leq \mathrm{p}_{i}^{L}, \\
L_{i, j}^{T}, & \text { otherwise }
\end{array}\right.
$$


where $L_{i, j}^{T}$ is the $j^{\text {th }}$ dimension of student $i$ 's behavior vector in generation $t$, with $i \in\{1,2,3 \ldots ., m\}$ and $j \in\{1,2,3 \ldots ., n\}, \Delta L_{i, j}^{T+1}$ is the behavior correction. Taking into account that the desire to learn from better people in a society will vary from person to person (typically better people are less likely to learn from the others), we describe a learning probability $p_{i}^{L}$ of each student $i$. Probability generated randomly $p_{i}$ satisfies $0 \leq \mathrm{p}_{i} \leq \mathrm{p}_{i}^{L} \leq 1$. Computationally, the following information is provided by a student:

Each student(S) in each section gains knowledge of their relevant Section Topper (ST) as follows:

$$
\Delta L_{i, j}^{T+1}=\phi_{1} \cdot \Delta L_{i, j}^{T+1}+\phi_{2} \cdot I^{T},
$$

with

$$
I^{T}=(S T-S)
$$

As a result, ST is the best student inside the section. The ST gains knowledge from the CT's knowledge. The following expressions:

$$
\Delta L_{i, j}^{T+1}=\phi_{1} \cdot \Delta L_{i, j}^{T+1}+\phi_{3} \cdot \varepsilon \cdot C^{T},
$$

with

$$
C^{T}=(C T-S T)
$$

The behavior correction $\Delta L^{T+1}$ consists of three components within the above modified frameworks influenced by social learning. In the CTO, the first factor $\Delta L^{T+1}$ is the same as the inertia component, while other components vary from Eq. (6) and Eq. (8). From Eq. (6), each learner in each section learns with their appropriate ST. Since this element is affected by imitation behaviour with normal social learning, it is referred to as the $\left(I^{T}\right)$ imitation part. Form Eq. (8), ST rises to become the section's top student. The ST learns from the CT. It learns out of the whole class's collective actions, i.e. all students' behavior. As the social impact factor $\left(C^{T}\right)$, the control parameter $\varepsilon$ is denoted. For simplicity, three random coefficients $\phi_{1}, \phi_{2}$ and $\phi_{3}$ have been replaced with the current parameter in $\mathrm{CTO}(w, n 1$ and $n 2)$ which will be generated randomly with $[0,1]$ once the updated strategy is executed.

There are three parameters to be defined in the proposed SL-CTO, i.e. the Student size $m$, the learning probability $p_{i}^{L}$ and the social influence factor $\varepsilon$. Student size $m$ is the first variable to be specified. It is suggested that the student size $m$ be calculated in the following form as a function of the search dimensional space [37]:

$$
m=M+\left|\frac{n}{10}\right|,
$$

Here $M$ was its basic student size for both the correct functioning of the SLCTO. The idea of a second parameter that sets the probability of learning $p_{i}^{L}$ is also inspired by natural social learning.

The following learning probability was adopted: 


$$
p_{i}^{L}=\left(1-\frac{i-1}{m}\right)^{\alpha \cdot \log \left(\left|\frac{n}{M}\right|\right)},
$$

where $\left(1-\frac{i-1}{m}\right)$ suggests that in a sorted student, the learning likelihood is inversely proportional to the $i$ performance index, $\alpha \cdot \log \left(\left|\frac{n}{M}\right|\right)$ specifies also that probability of learning is inversely related to the complexity of a search, and $\alpha \cdot \log (\cdot)$ the admixed feature is used to smooth the effect of $\frac{n}{M}$. Empirically, the coefficient $\alpha<1$ is recommended, and also in the said work, $\alpha=0.6$ was used. If the probability of learning meets $p_{i}(t) \leq p_{i}^{L}, L_{i, j}^{T}$ will be corrected as follows:

$$
L_{i, j}^{T+1}=L_{i, j}^{T}+\Delta L_{i, j}^{T+1},
$$

The following expression can be obtained, if we substitute Eq. (6) and Eq. (8) for Eq. (7) and Eq. (9) respectively, and replace all random parameters with their predicted value:

$$
\begin{gathered}
\Delta L_{i, j}^{T+1}=\frac{1}{2} \cdot \Delta L_{i, j}^{T+1}+\frac{1}{2} \cdot(S T-S), \\
\Delta L_{i, j}^{T+1}=\frac{1}{2} \cdot \Delta L_{i, j}^{T+1}+\frac{1}{2} \cdot \varepsilon \cdot(C T-S T),
\end{gathered}
$$

where $1 / 2$ is the predicted value of $\phi_{1}, \phi_{2}$ and $\phi_{3}$.

The last variable that is left to define is the social impact variable $\varepsilon$. The convergence complexity is generally proportional to the dimensional space of the search, since the convergence of the entire class involves the convergence for each dimension into each student's functional vector. The social influence factor $\varepsilon$ is described on the basis of this observation as follows:

$$
\varepsilon=\beta \times \frac{n}{M},
$$

That means $\varepsilon$ is inversely related to the dimension of the problem. Since the effect of the class-level mean behaviour is controlled, if the value of this variable has also been set too high, premature integration with the mean behaviour (instead of the best behaviour) could occur. In our work, a smaller value for $\beta=0.01$ is therefore used.

In this way, we may reduce the convergence of the proposed SL-CTO to the convergence of our model.

4.1 Description of Proposed enhanced correction factor DV-Hop localization algorithm using SL - CTO

In this section, a new SL - CTO based, enhanced rectification factor DV-Hop localization algorithm that includes the following steps: 


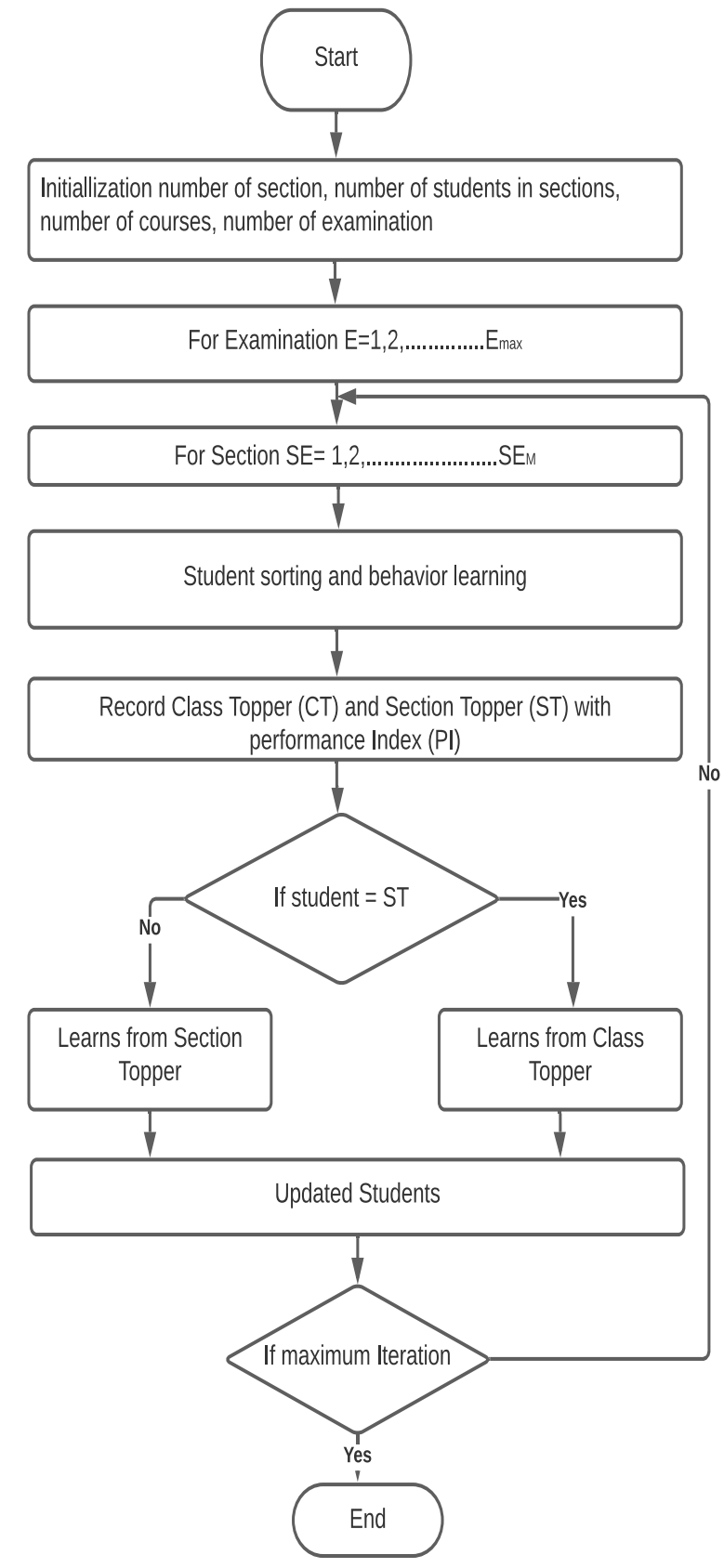

Fig. 1: Flowchart of social learning class topper optimization algorithm. 
Step 1 Calculate the beacon node coordinates and relay to the network the position of the nodes. The minimum hop count value will be obtained by all sensor nodes in the network after that.

Step 2 The dumb node defines the modified hop size for the beacon nodes based on the number of hops, wight of beacon node [38] as well as distance information among beacon nodes as shown below:

$$
j p_{i}^{\bmod }=\frac{j p_{i}}{w},
$$

where the weight of beacon node is $w$.

The approximate difference between the $i$ and $j$ beacon nodes is calculated as

$$
p_{i j}^{\text {appro }}=j p_{i}^{\bmod } \times h o p_{i j} .
$$

The true distance between beacon nodes $i$ and $j$ shall be calculated by

$$
p_{i j}^{\text {true }}=\sqrt{\left(a_{i}-a_{j}\right)^{2}+\left(b_{i}-b_{j}\right)^{2}} .
$$

Error between nodes $i$ and $j$ of the beacon is given as

$$
p_{i j}^{\text {error }}=p_{i j}^{\text {appro }}-p_{i j}^{\text {true }} .
$$

Now, we have added a rectification factor, and this is defined as:

$$
\tau=\frac{p_{i j}^{\text {error }}}{s}
$$

where $s$ is the number of beacon nodes.

The $\tau$ rectification factor is used by adding it to the previous hop size to change the hop size of the beacon node.

The shifted distance between the $i$ beacon nodes and the $k$ dumb node is determined as [37]:

$$
p_{i k}^{M o d}=(H o p S i z e+\tau) \times h o p_{i k} .
$$

Step 3 To calculate the location of dumb nodes, the $2 D$ hyperbolic position technique is used. Let $(a, b)$ be the location of the dumb node and $a_{i}, b_{i}$ be the position of the $i^{t h}$ beacon node. The following formula is used to calculate the distance between these nodes:

$$
p_{i}=\sqrt{\left(a_{i}-a\right)^{2}+\left(b_{i}-b\right)^{2}} .
$$

The following formula can be used to calculate this:

$$
p_{i}^{2}=\left(a_{i}-a\right)^{2}+\left(b_{i}-b\right)^{2},
$$

where $S_{i}=a_{i}^{2}+b_{i}^{2}$ and $T=a^{2}+b^{2}$,

$$
p_{i}^{2}-S_{i}=-2 a_{i} a-2 b_{i} b T .
$$


Suppose

$$
\begin{aligned}
& Z=[a, b, T]^{T},
\end{aligned}
$$

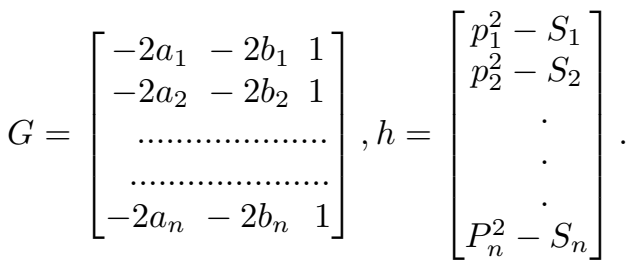

Finally, with the aid of the least mean square estimated process, we can gain $Z$ value:

$$
Z=\left(G^{T} G\right)^{-1} G^{T} h .
$$

Step 4 To correct this approximate position of the dumb nodes, we used the SL - CTO algorithm. The objective function of SL - CTO using DV-Hop localization is formulated mathematically as:

$$
f(a, b)=\min \left[\sum_{i=1}^{S}\left|\sqrt{\left(a-a_{i}\right)^{2}+\left(b-b_{i}\right)^{2}}-p_{i k}^{M o d}\right|\right]
$$

The flow chart for the proposed localization algorithm is shown in Fig. 2.

\section{Model of communication imbalance}

In actual life, the communication pattern of sensor nodes is not similar in every direction. An unusual broadcast path can be caused by various propagation loss in RF transmission data. In the network of wireless sensors, communication disturbances are a common issue. So we considered a complete situation in this paper for formulating modulus of elasticity and discovering the effect of communication imbalance on our proposed algorithm of localization. To indicate that irregularity of a communication signal, the variable DOI ( degree of irregularity) is inserted into to the model. The DOI variable is based on the maximum propagation loss ratio variance per unit degree shift in direction for communication networks. VSP (Sending Power Variance), which will be attributed to the high percentage variance between the multiple devices of the transmission sending power. We modeled the distortion of the communication method used in the following equation [37]:

$$
C_{f}=C \times(1-\text { Imbalance Variable } \times \text { gamma }),
$$

where $C_{f}$ is the maximum range of transmission after an imbalance variable is imposed, $C$ is the effective transmission range before an imbalance variable is imposed, $\gamma$ is uniformly dispersed by an unique number identified as: $\gamma \sim$ $U(0,1)$ and the factor of irregularity is any amount that is $\in[0,0.5]$. 


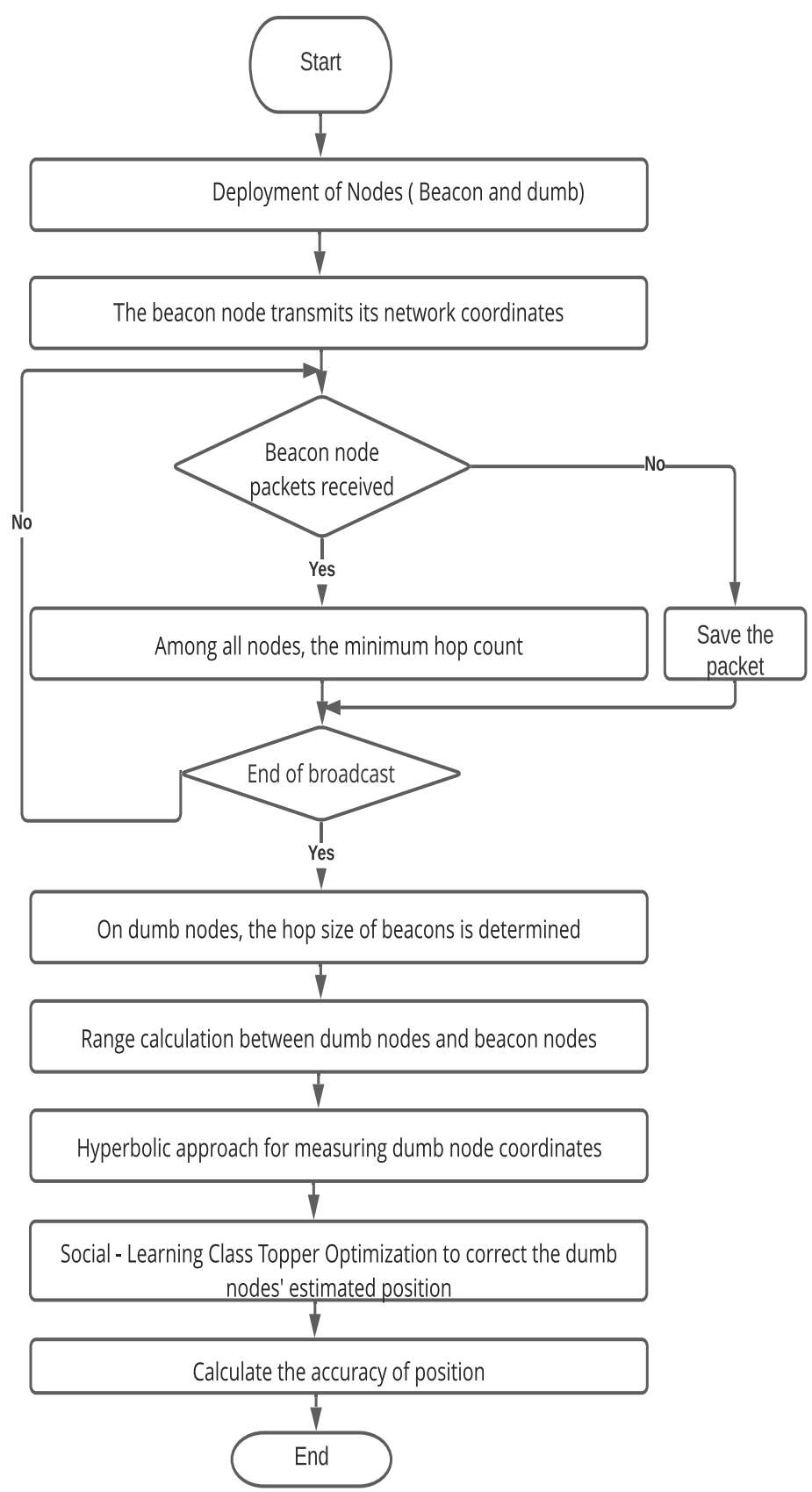

Fig. 2: Flowchart of proposed algorithm. 


\section{Metrics for Performance}

The efficacy of the suggested algorithm was as determined by the basis of time for computation, error in localization and error variance in localization, as follows:

\subsection{Time for computation}

Computation time is the amount of time taken during the entire localization process to perform a computational process. It is calculated using the tictoc function. We contrasted the computation time for suggested algorithm in this paper with original DV-Hop [17], PSO-based DV-Hop [22] and RRA-based DV-Hop [6] localization algorithms.

\subsection{Error in localization}

The measurement of the dumb node's true position resulted in a location error. The total number of dumb nodes, beacon nodes, imbalance variable, communication range, and distribution area all had an effect on the localization error. For localization, the average error is calculated as:

$$
A L E=\frac{\sum_{i=1}^{t} \sqrt{\left(a^{\text {appro }}-a^{t r}\right)^{2}+\left(b^{\text {appro }}-b^{t r}\right)^{2}}}{t \times R^{2}},
$$

where $t$ represents the amount of dumb nodes and $R$ represents the sensor node's communication range.

6.3 Variance of localization error

The variance is a measure of how each amount is derived from the mean in the set:

$$
L E V=\frac{\sum_{i=1}^{t}\left(A L E_{i}-A L E_{s}\right)^{2}}{t},
$$

where $A L E_{s}$ is the mean of error of localization. 


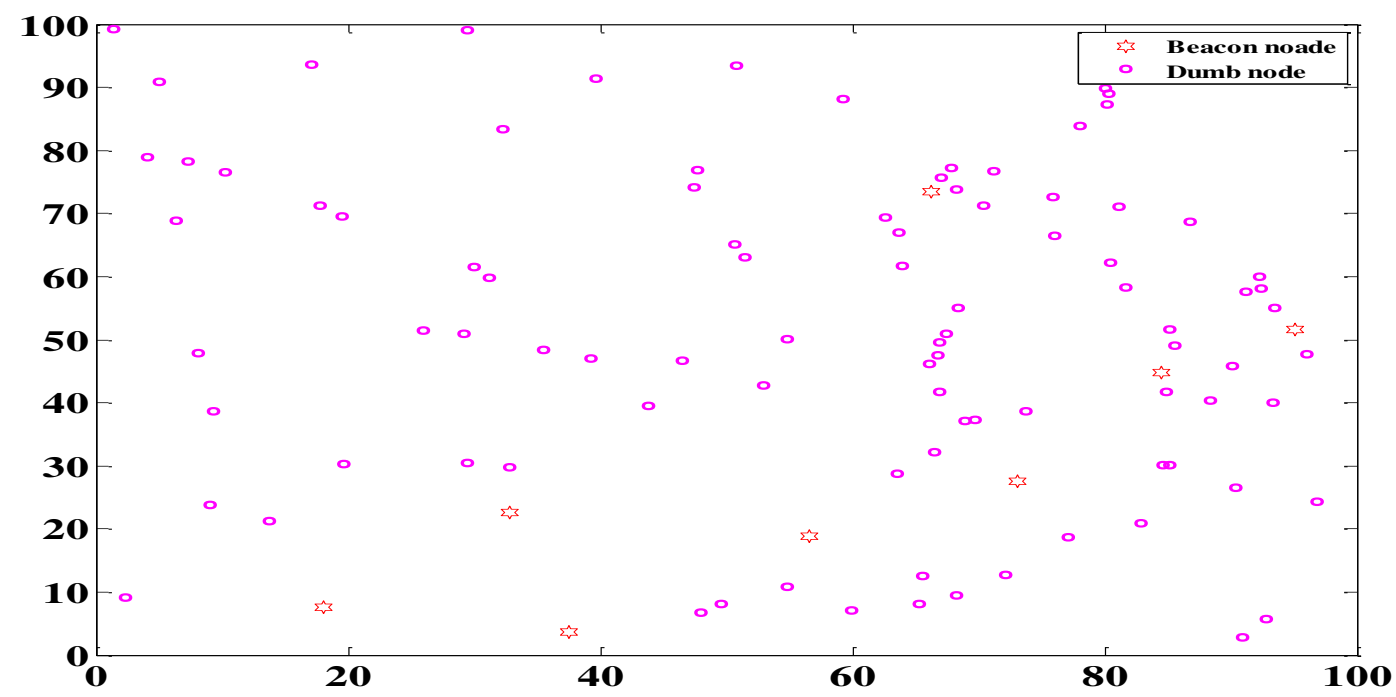

Fig. 3: Uniformly distributed dumb and beacon nodes.

Table 1: Parameters for simulation.

\begin{tabular}{|l|l|}
\hline Parameter & Value \\
\hline WSN area & $100 * 100 \sim 300 * 300 \mathrm{~m}^{2}$ \\
\hline Number of dumb nodes $(\mathrm{t})$ & $100 \sim 400$ \\
\hline Number of beacon nodes $(\mathrm{S})$ & $20 \sim 160$ \\
\hline Communication range & $15 \sim 35$ \\
\hline Imbalance variable & $0.1 \sim 0.45$ \\
\hline Maximum iterations & 30 \\
\hline Algorithm for optimization & $\mathrm{SL}-\mathrm{CTO}$ \\
\hline$\phi_{1}, \phi_{2}$ and $\phi_{3}$ & 0.5 \\
\hline$\epsilon_{\text {socialinfluencefactor }}$ & $>-1$ \\
\hline$\beta$ & 0.01 \\
\hline
\end{tabular}

\section{Simulations and Outcomes}

Using LabVIEW@2015,We looked into the effectiveness of the offered method. This part provides an analysis of performance in definitions of localization error, localization error variance and computation time. The simulation outcomes assessed the quality of the suggested algorithm for various parameter alterations, i.e. node size, beacon size, communication range, irregularity factor and distribution area. The parameters of the simulation are summarized in Table 1. For simulation purposes, We considered a region of $100 \times 100 \mathrm{~m}^{2}$ with dumb and beacon nodes uniformly distributed, as illustrated in Fig. 3. 


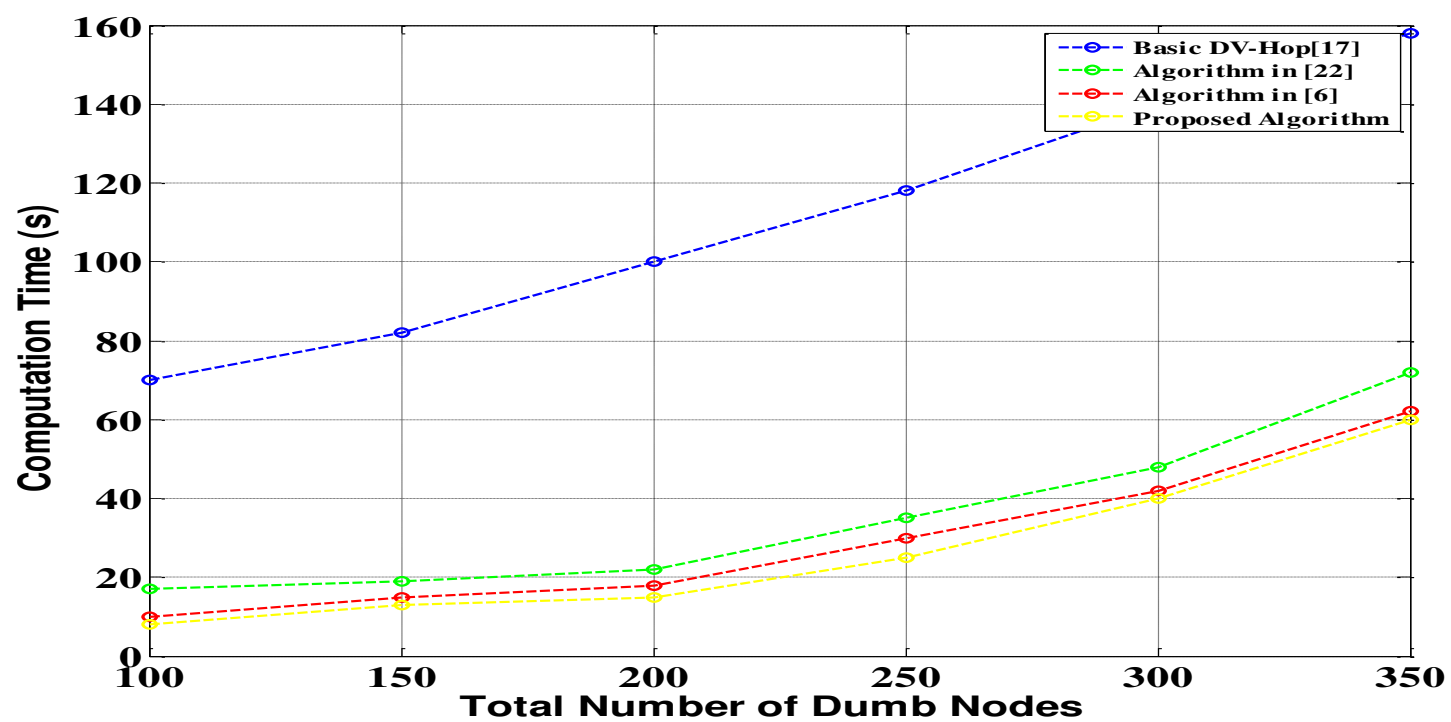

Fig. 4: Computation time variance with dumb nodes.

Table 2: A comparison of the suggested algorithm's effect on the overall number of dumb nodes resulting from computation time vs several current approaches.

\begin{tabular}{|l|l|l|l|l|l|l|}
\hline Total no of dumb nodes & 100 & 150 & 200 & 250 & 300 & 350 \\
\hline Proposed & .20 & .13 & .16 & .16 & .04 & .032 \\
\hline RRA DV Hop [6] & .25 & .15 & .2 & .2 & .05 & .03 \\
\hline PSO DV Hop [22] & 1.12 & .46 & .46 & .4 & .2 & .2 \\
\hline Basic DV Hop [17] & 7.75 & 5.3 & 5.6 & 3.72 & 2.5 & 1.6 \\
\hline
\end{tabular}

\subsection{Effect on computation time of the total number of dumb nodes}

The computation time difference as the number of dumb nodes changes is given in Fig. 4. An region of $100 \times 100 \mathrm{~m}^{2}$ is considered to have 25 beacon nodes and $25 \mathrm{~m}$ of transmission range fixed for simulation. The number of dumb nodes varies from 100 to 400. Calculation time has been shown to increase as the overall number of dumb nodes increases. It can be observed from Table 2 that, compared to PSO DV-Hop and RRA DV-Hop, our algorithm needs less computational time.

\subsection{Effect on computation time of the total number of beacon nodes}

The difference throughout computation time caused by a change in the amount of beacon nodes is illustrated in Fig. 5. An region of $100 \times 100 \mathrm{~m}^{2}$ is considered 


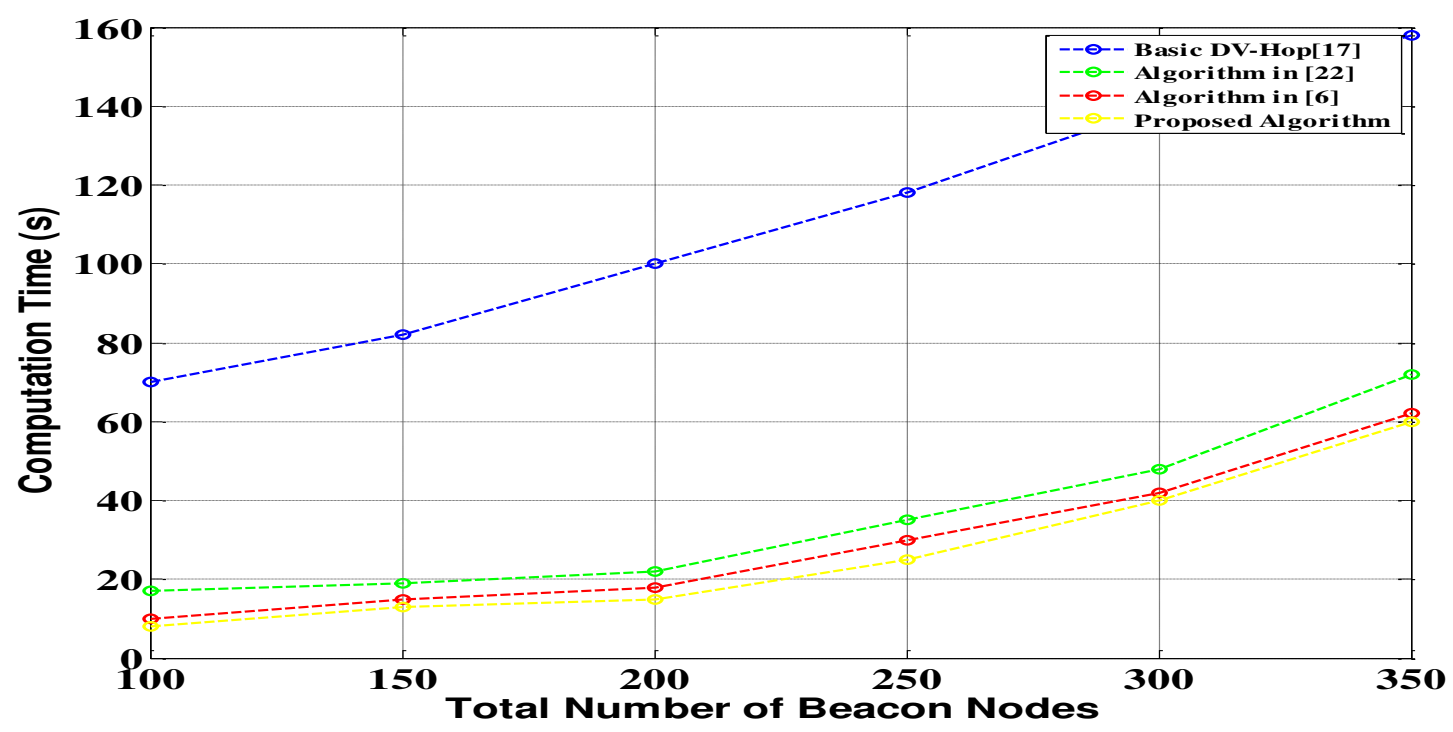

Fig. 5: Variation in the time of computation with beacon nodes.

Table 3: A comparison of the proposed algorithm's effect on the overall number of beacon nodes based on computation time with various current algorithms is being conducted.

\begin{tabular}{|l|l|l|l|l|l|l|}
\hline Total no of beacon nodes & 20 & 52 & 78 & 100 & 122 & 150 \\
\hline Proposed & .14 & .15 & .04 & .034 & .046 & .024 \\
\hline RRA DV Hop [6] & .16 & .18 & .05 & .03 & .04 & .02 \\
\hline PSO DV Hop [22] & .58 & 1 & .45 & .10 & .27 & .06 \\
\hline Basic DV Hop [17] & 3.5 & 2.6 & 2.05 & 1.46 & 1.36 & 0.97 \\
\hline
\end{tabular}

to have 200 dumb nodes and a $25 \mathrm{~m}$ communication range fixed for simulation. The beacon nodes range between 20 and 160. Table 3 indicates that with an increase in the number of beacon nodes, the calculation time increases.

7.3 Effect of the overall amount of dumb nodes on localization error and variance

Out of Fig. 6 and Fig. 7 localization error and variance were found to decrease with an enhance in the number of dumb nodes. An region of $100 \times 100 \mathrm{~m}^{2}$ is regarded to have 30 beacon nodes and $25 \mathrm{~m}$ of transmission range fixed for simulation. The number of dumb nodes varies from 100 to 400. It is noted in Table 4 that the basic DV-Hop method has much less localization accuracy likened to the our method. 


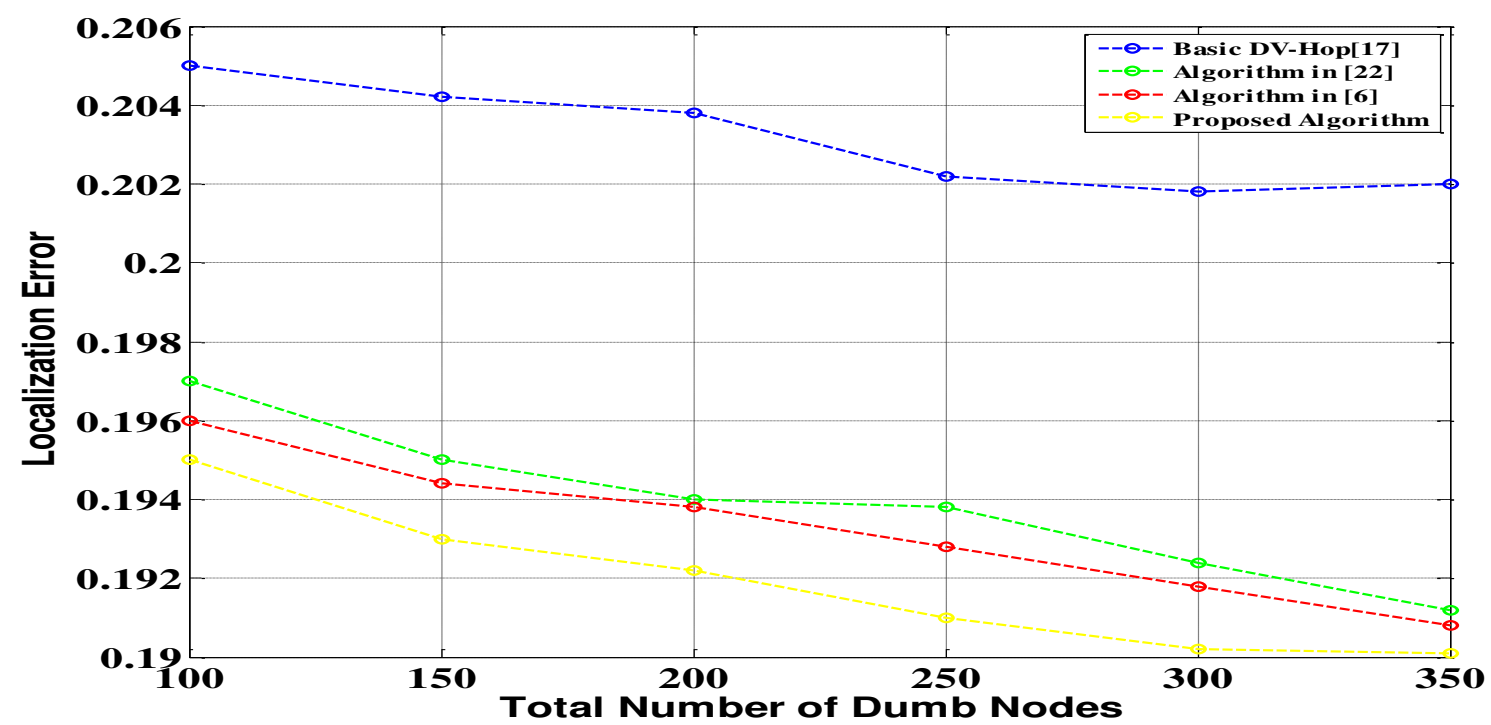

Fig. 6: With dumb nodes, there is a variation in the localization error.

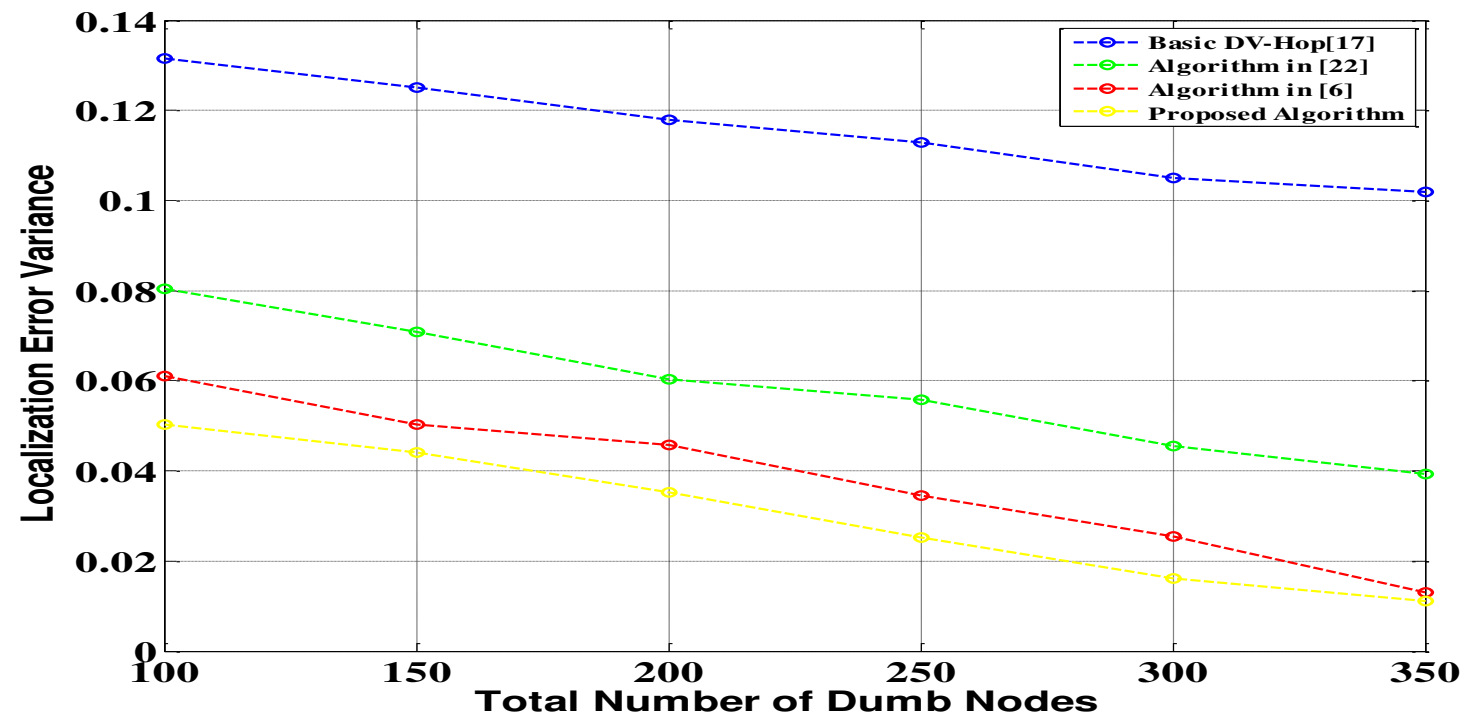

Fig. 7: change in a localization error variance with dumb nodes. 
Table 4: A comparison of the proposed method's effect on the overall amount of dumb nodes from LE and LEV with the effects of other techniques.

\begin{tabular}{|l|l|l|l|l|l|l|l|}
\hline Total Number of dumb nodes & & 100 & 150 & 200 & 250 & 300 & 350 \\
\hline \multirow{3}{*}{ Proposed } & LE & .0051 & .0072 & .0082 & .0093 & .0083 & .0034 \\
\cline { 2 - 9 } & LEV & .072 & .02 & .041 & .042 & .003 & .001 \\
\hline \multirow{3}{*}[6]{} & LE & .0052 & .007 & .0083 & .009 & .0084 & .0036 \\
\cline { 2 - 9 } & LEV & .078 & .03 & .043 & .043 & .004 & .002 \\
\hline \multirow{2}{*}{ PSO-DV-Hop } & LE & .010 & .014 & .0093 & .014 & .011 & .005 \\
\cline { 2 - 9 } & LA & .107 & .08 & .10 & .032 & .061 & .043 \\
\hline \multirow{2}{*}[17]{} & LE & .05 & .058 & .06 & .05 & .06 & .06 \\
\cline { 2 - 9 } & LA & 11.8 & 0.25 & 0.26 & 0.24 & .22 & .16 \\
\hline
\end{tabular}

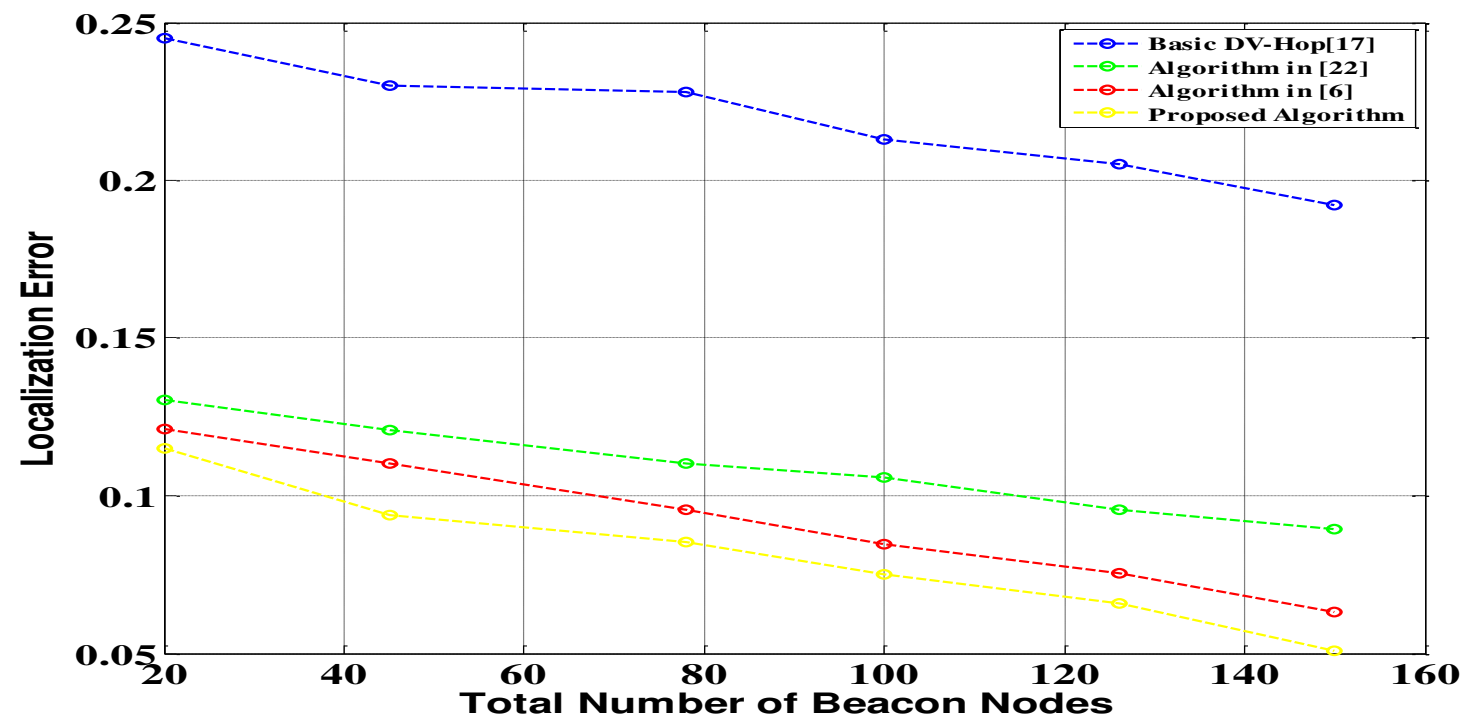

Fig. 8: Variation of a difference in a localization error with beacon nodes.

7.4 Effect of the overall number for beacon nodes on localization error and variance

Fig. 8 and 9 values indicate the impact of the total number of beacon nodes on the localization error and variance. The $100 \times 100 \mathrm{~m}^{2}$ area is considered to have fixed 200 dumb nodes and a communication range of $25 \mathrm{~m}$ for the simulation,beacon nodes range from 20 to 160 . It is noted in the Table 5 that, compared to RRA DV-Hop, PSO DV-Hop and conventional DV-Hop, the proposed SL - CTO DV-Hop has improved localization accuracy. 


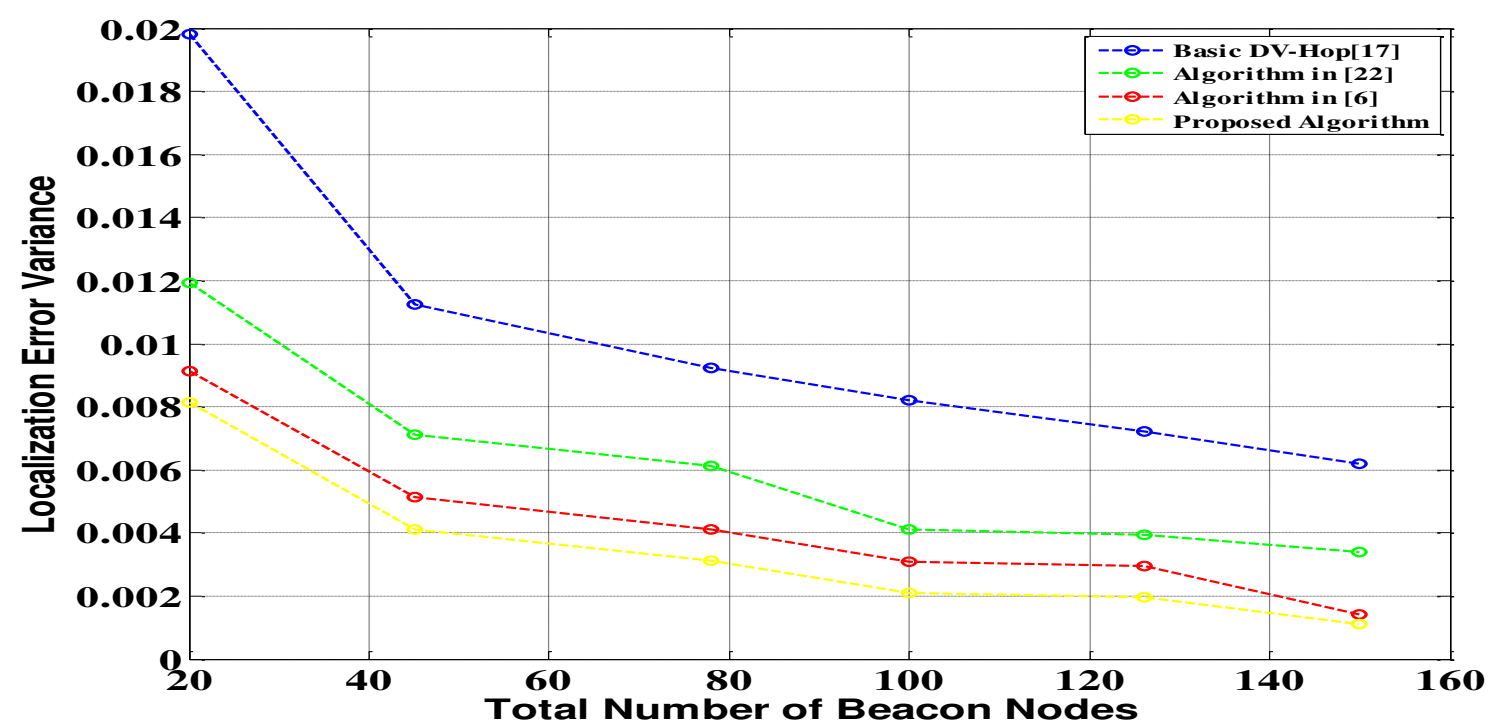

Fig. 9: Changes in the difference in a localization error of beacon nodes.

Table 5: A comparison of the proposed method's effect on the overall amount of beacon nodes between LE and LEV with the effects of other algorithms.

\begin{tabular}{|l|l|l|l|l|l|l|l|}
\hline Total Number of beacon nodes & & 20 & 45 & 78 & 100 & 126 & 150 \\
\hline \multirow{3}{*}{ Proposed } & LE & .070 & .053 & .05 & .03 & .02 & .045 \\
\cline { 2 - 8 } & LEV & .11 & .006 & .21 & .29 & .01 & .12 \\
\hline \multirow{3}{*}{ RRA-DV-Hop } & LE & .075 & .056 & .06 & .04 & .04 & .047 \\
\cline { 2 - 8 } & LEV & .12 & .10 & .27 & .42 & .03 & .14 \\
\hline \multirow{2}{*}{ Basic DV-Hop } & LE & .133 & .12 & .14 & .12 & .13 & .14 \\
\cline { 2 - 8 } & LEV & .33 & .43 & .81 & .73 & .40 & .52 \\
\hline \multirow{2}{*}[17]{} & LE & .44 & .61 & .80 & .76 & .80 & .85 \\
\cline { 2 - 8 } & LEV & 1.2 & 1.9 & 3.6 & 4.5 & 2.8 & 3.8 \\
\hline
\end{tabular}

7.5 Effect of communication range on error of localisation and variation of localization error

The effect of communication range on localization error and variance are illustrated in Fig. 10 and 11. An region of $100 \times 100 \mathrm{~m}^{2}$ for simulation. The units are considered to have 300 dumb nodes or unknown nodes and 25 beacon nodes fixed. The transmission ranges between 15 and 35 metres. The simulation results in Table 6 show that as communication range is increased, localization error and variance decreases. 


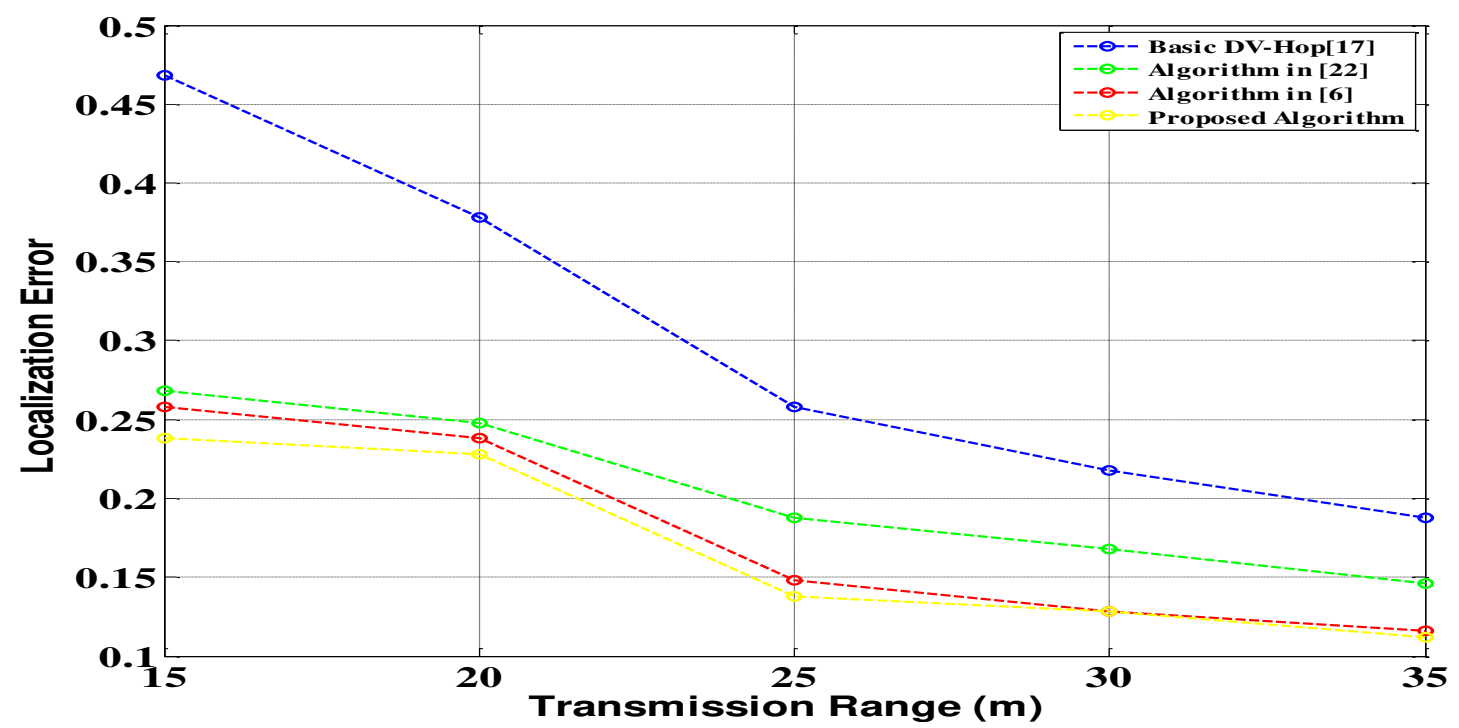

Fig. 10: Changes in a localization error as a function of transmission range.

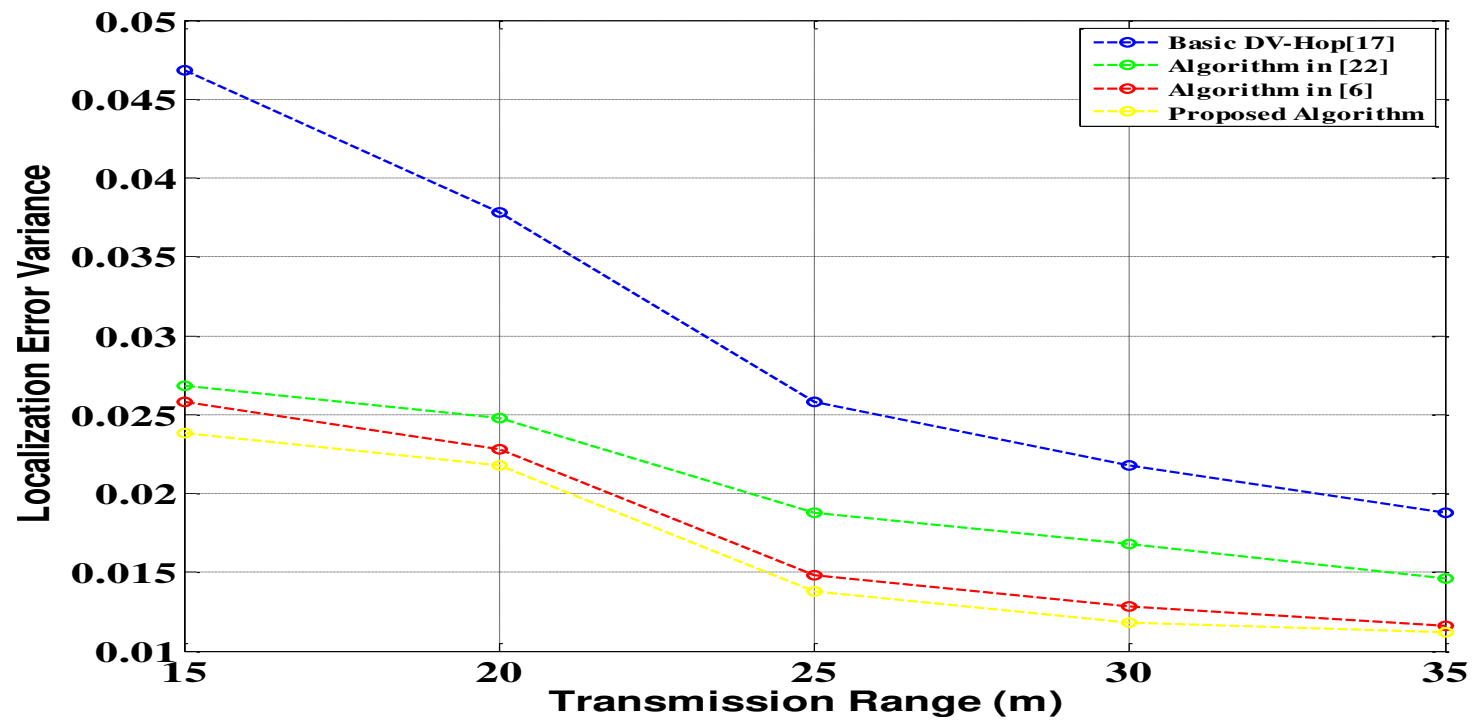

Fig. 11: Change in the transmission range of a localization error. 
Table 6: A comparison of the suggested algorithm's effect on transmission range from LE and LEV compared to some current algorithms.

\begin{tabular}{|l|l|l|l|l|l|l|}
\hline Transmission Range (m) & & 15 & 20 & 25 & 30 & 35 \\
\hline \multirow{3}{*}{ Proposed } & LE & .033 & .02 & .031 & .21 & .035 \\
\cline { 2 - 7 } & LEV & .01 & .056 & .11 & .15 & .25 \\
\hline \multirow{2}{*}[6]{} & LE & .049 & .05 & .032 & .37 & .043 \\
\cline { 2 - 7 } & LEV & .03 & .076 & .22 & .28 & .33 \\
\hline PSO-DV-Hop & LE & .21 & .23 & .16 & .18 & .28 \\
\cline { 2 - 7 } Basic DV-Hop & LEV & .24 & .35 & .26 & .6 & .66 \\
\cline { 2 - 7 } & LE & .89 & 1.04 & .88 & 1 & 1 \\
\cline { 2 - 7 } & LEV & 1.4 & 3.6 & 4.6 & 5.8 & 6 \\
\hline
\end{tabular}

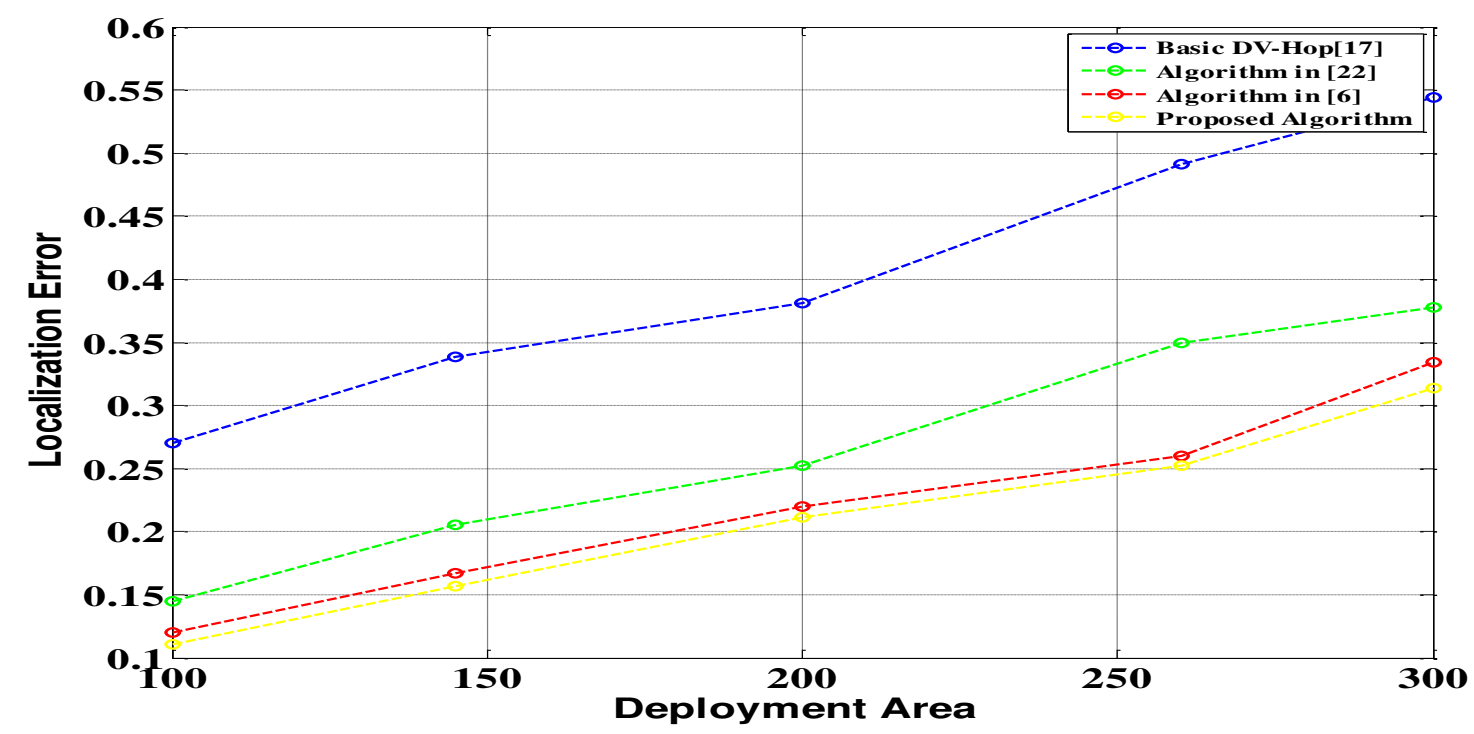

Fig. 12: Variation for a deployment area localization error.

7.6 Effect for area of deployment on error in localization and variance error in localization

The effect for deployment region on localization error and variance is depicted in Fig. 12 and 13 . Set 300 dumb nodes, 50 beacon nodes and $30 \mathrm{~m}$ of communication range are assumed for simulation. The area of deployment ranges from $100 * 100 \sim 300 * 300 \mathrm{~m}^{2}$. With an increased distribution area, localization error has been observed to increase. In addition, it can be seen from table 7 that, compared to conventional DV-Hop, PSO DV-Hop and RRA DV-Hop, the proposed method has less localization error and variance. 


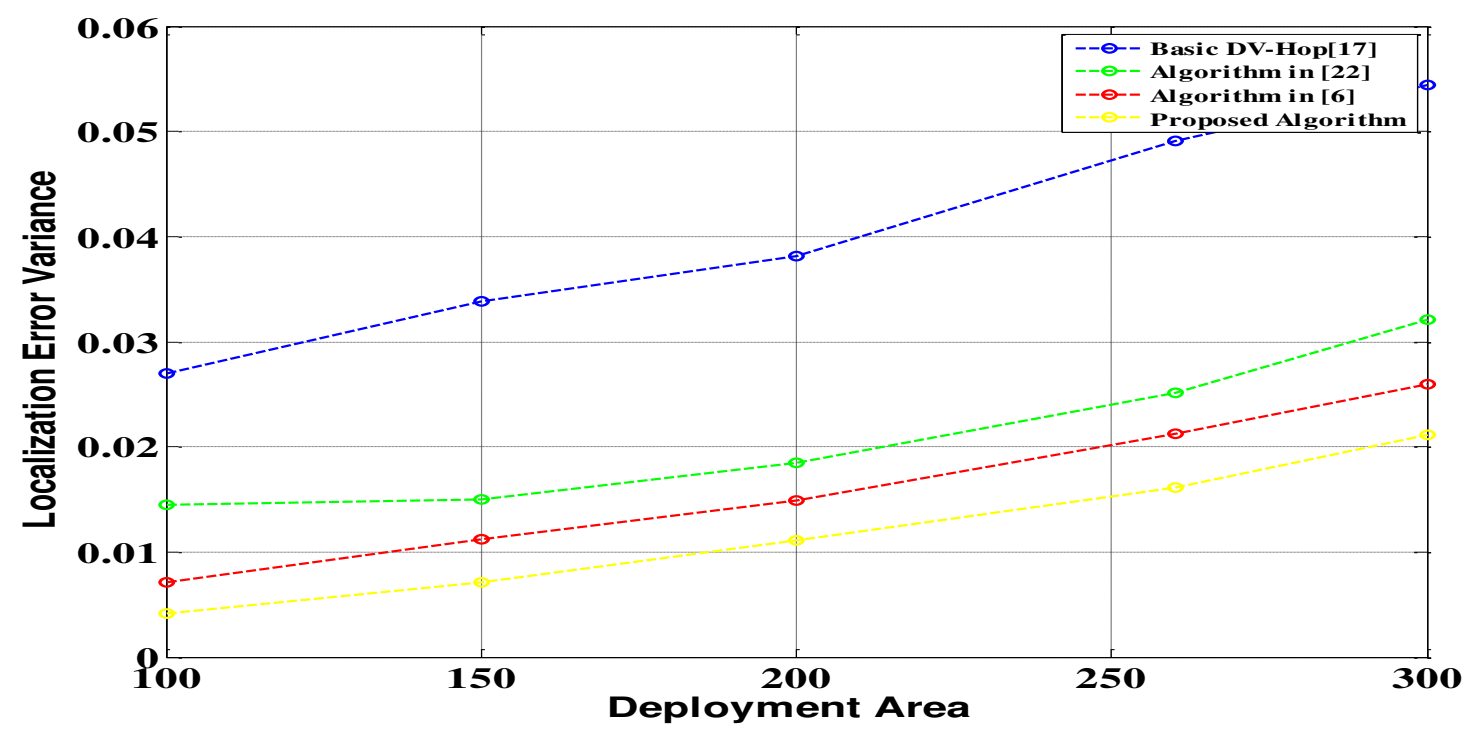

Fig. 13: Variation for a deployment area localization error variance.

Table 7: A comparison of the proposed method's effect on the deployment area from LE and LEV with several current algorithms.

\begin{tabular}{|l|l|l|l|l|l|l|}
\hline Deployment Area & & 100 & 145 & 200 & 250 & 300 \\
\hline \multirow{3}{*}{ Proposed } & LE & .06 & .03 & .01 & .02 & .045 \\
\cline { 2 - 7 } & LEV & .11 & .05 & .02 & .015 & .045 \\
\hline \multirow{2}{*}{ RRA-DV-Hop } & LE & .09 & .05 & .02 & .04 & .048 \\
\cline { 2 - 7 } & LEV & .14 & .07 & .04 & .027 & .06 \\
\hline \multirow{2}{*}[22]{} & LE & .31 & .048 & .16 & .17 & .048 \\
\cline { 2 - 7 } Basic DV-DV-Hop & LEV & 1 & .69 & .26 & .24 & .21 \\
\hline \multirow{2}{*}[17]{} & LE & 1.4 & 1.1 & .76 & .96 & .50 \\
\cline { 2 - 7 } & LEV & 4.8 & 2.9 & 1.7 & .88 & .95 \\
\hline
\end{tabular}

7.7 Effect for the variable of imbalance on localization error and variance of localization error

Fig. 14 and 15 shows the impact of the variable of imbalance on the variance of localization error and variance. An region of $100 \times 100 \mathrm{~m}^{2}$. for simulation. With 200 dumb nodes, 25 beacon nodes and $25 \mathrm{~m}$ communication range fixed, units are considered. The imbalance variable ranges between 0.1 and 0.45 . It was discovered from Table 8 that localization error increases with an improvement in the imbalance variable. 


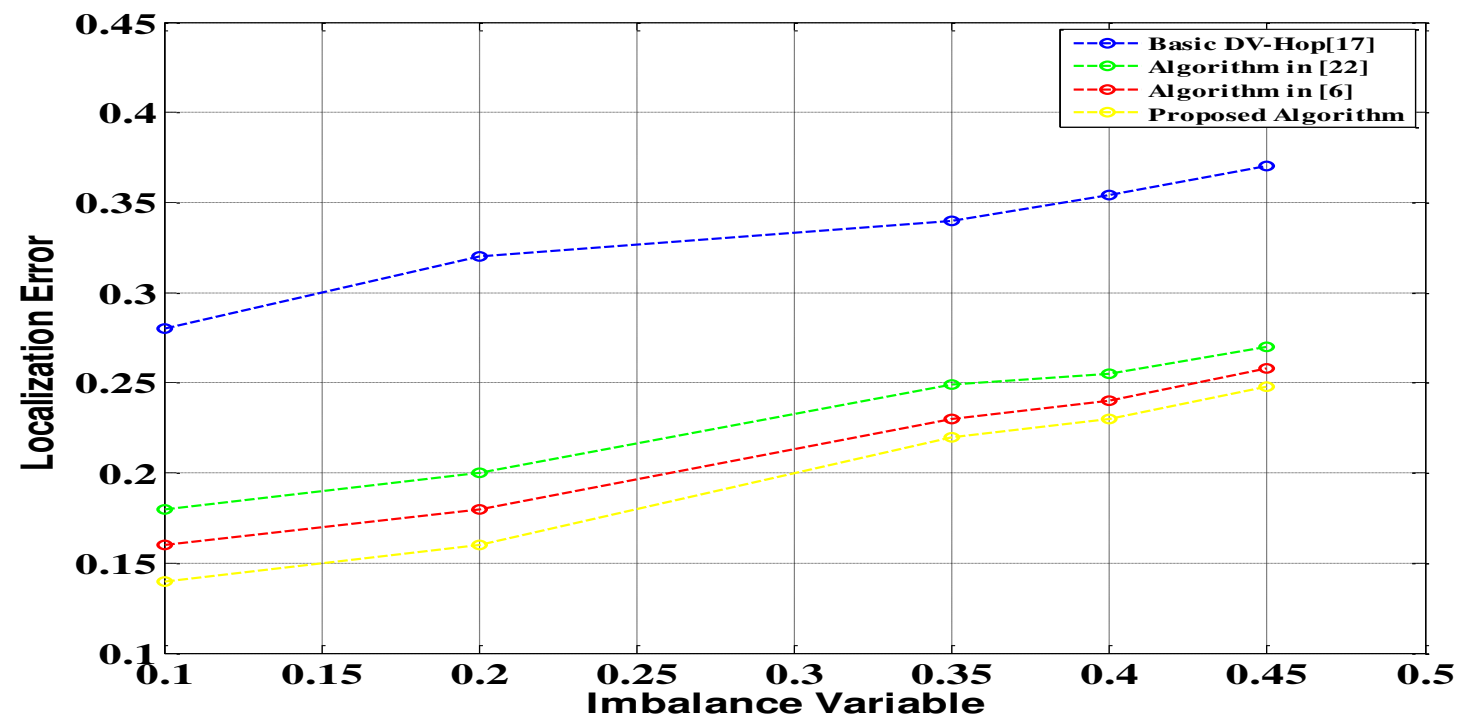

Fig. 14: Variation in a localization error with imbalance variable.

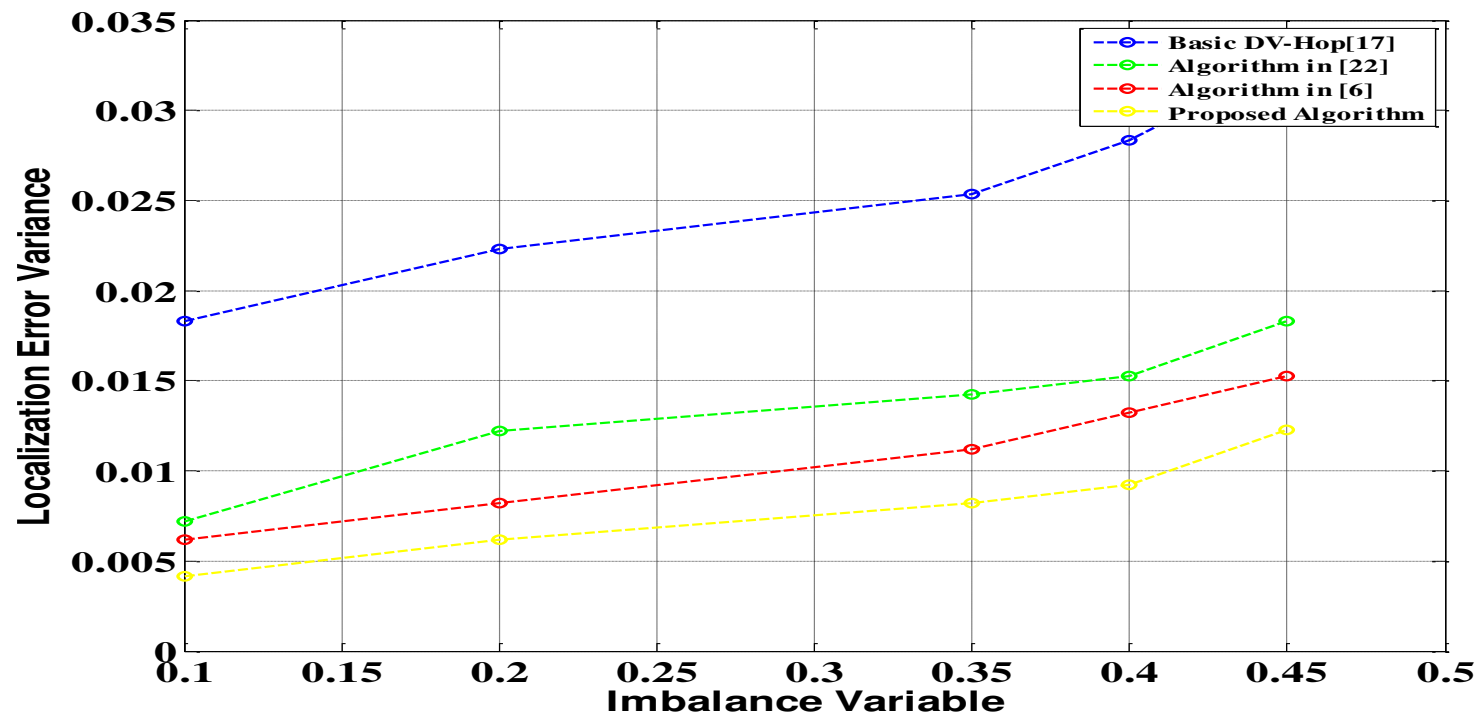

Fig. 15: Change in a localization error variance with imbalance variable. 
Table 8: A comparison of the suggested algorithm's effect on imbalance variables from LE and LEV with several current algorithms was conducted.

\begin{tabular}{|l|l|l|l|l|l|l|}
\hline Irregularity factor & & .1 & .2 & .3 & .4 & .45 \\
\hline \multirow{3}{*}{ Proposed } & LE & .03 & .06 & .0115 & .07 & .11 \\
\cline { 2 - 7 } & LEV & .21 & .15 & .125 & .03 & .145 \\
\hline \multirow{2}{*}[6]{} & LE & .06 & .08 & .013 & .10 & .125 \\
\cline { 2 - 7 } & LEV & .28 & .2 & .14 & .05 & .18 \\
\hline \multirow{2}{*}{ PSO-DV-Hop $[22]$} & LE & .12 & .20 & .23 & 1.09 & 1 \\
\cline { 2 - 7 } & LEV & .65 & .4 & .71 & .4 & .45 \\
\hline \multirow{2}{*}{ Basic DV-Hop } & LE & .80 & .41 & .85 & 2.12 & 1.9 \\
\cline { 2 - 7 } & LEV & 4.14 & 3.2 & 2.5 & 1.52 & 1.8 \\
\hline
\end{tabular}

\section{Conclusion}

We presented a DV-Hop localization based on a correction factor based on social learning class topper optimization (SL-CTO) in this work to reduce localization error and increase precision in positioning. We evaluated the hop size for the beacons at the dumb nodes in the suggested technique. This reduces full communication between beacon and dumb nodes, making the proposed algorithm energy efficient. The correction factor is used to change the hop size of the beacon nodes. It is also considered that the model of network imbalance illustrates the applicability of the proposed anisotropic network algorithm. Simulation results demonstrate that, relative to conventional localization methods, the proposed algorithm performs better calculation time, localization error and variance. The location of the node in the wireless sensor networks is also an important framework. Energy consumption and the time required for the future path are two other important factors to consider in the proposed algorithm. One of the future research projects would be to apply the Social Learning Class Topper Optimization (SL-CTO)- Hop in a three-dimensional framework and on a real proving ground.

\section{References}

[1] R. Kumar, S. Kumar, D. Shukla, R. S. Raw, and O. Kaiwartya, "Geometrical localization algorithm for three dimensional wireless sensor networks," Wireless personal communications, vol. 79, no. 1, pp. 249-264, 2014.

[2] G. Kumar, M. K. Rai, R. Saha, and H.-j. Kim, "An improved dv-hop localization with minimum connected dominating set for mobile nodes in wireless sensor networks," International Journal of Distributed Sensor Networks, vol. 14, no. 1, p. $1550147718755636,2018$.

[3] H. Rashid and A. K. Turuk, "Localization of wireless sensor networks using a single anchor node," Wireless personal communications, vol. 72, no. 2, pp. 975-986, 2013.

[4] M. Jiang, Y. Li, Y. Ge, W. Gao, K. Lou, S. Wang, and J. Jiang, "Improved dv-hop localization algorithm based on anchor weight and distance compensation in wireless sensor network," International Journal of Signal Processing, Image Processing and Pattern Recognition, vol. 9, no. 12, pp. 167-176, 2016.

[5] V. Gupta and B. Singh, "Study of range free centroid based localization algorithm and its improvement using particle swarm optimization for wireless sensor networks 
under log normal shadowing," International Journal of Information Technology, pp. $1-7,2018$.

[6] V. Kanwar and A. Kumar, "Dv-hop-based range-free localization algorithm for wireless sensor network using runner-root optimization," The Journal of Supercomputing, pp. $1-18,2020$.

[7] L. Gui, X. Zhang, Q. Ding, F. Shu, and A. Wei, "Reference anchor selection and global optimized solution for dv-hop localization in wireless sensor networks," Wireless Personal Communications, vol. 96, no. 4, pp. 5995-6005, 2017.

[8] S. Pandey and S. Varma, "A range based localization system in multihop wireless sensor networks: a distributed cooperative approach," Wireless Personal Communications, vol. 86, no. 2, pp. $615-634,2016$.

[9] M. Shalaby, M. Shokair, and N. W. Messiha, "Performance enhancement of toa localized wireless sensor networks," Wireless Personal Communications, vol. 95, no. 4, pp. 46674679, 2017.

[10] O. Oguejiofor, A. Aniedu, H. Ejiofor, and A. Okolibe, "Trilateration based localization algorithm for wireless sensor network," International Journal of Science and Modern Engineering (IJISME), vol. 1, no. 10, pp. 2319-6386, 2013.

[11] J. Kumari, P. Kumar, and S. K. Singh, "Localization in three-dimensional wireless sensor networks: a survey," The Journal of Supercomputing, vol. 75, no. 8, pp. 5040$5083,2019$.

[12] R. Shahbazian and S. A. Ghorashi, "Distributed cooperative target detection and localization in decentralized wireless sensor networks," The Journal of Supercomputing, vol. 73, no. 4, pp. 1715-1732, 2017.

[13] J. W. Park, D. H. Park, and C. Lee, "Angle and ranging based localization method for ad hoc network," The Journal of Supercomputing, vol. 64, no. 2, pp. 507-521, 2013.

[14] T. Najeh, H. Sassi, and N. Liouane, "A novel range free localization algorithm in wireless sensor networks based on connectivity and genetic algorithms," International Journal of Wireless Information Networks, vol. 25, no. 1, pp. 88-97, 2018.

[15] F. Mekelleche and H. Haffaf, "Classification and comparison of range-based localization techniques in wireless sensor networks," Journal of Communications, vol. 12, no. 4, pp. $221-227,2017$.

[16] J. Mass-Sanchez, E. Ruiz-Ibarra, J. Cortez-González, A. Espinoza-Ruiz, and L. A. Castro, "Weighted hyperbolic dv-hop positioning node localization algorithm in wsns," Wireless Personal Communications, vol. 96, no. 4, pp. 5011-5033, 2017.

[17] D. Niculescu and B. Nath, "Dv based positioning in ad hoc networks," Telecommunication Systems, vol. 22, no. 1-4, pp. 267-280, 2003.

[18] X. Chen and B. Zhang, "Improved dv-hop node localization algorithm in wireless sensor networks," International Journal of Distributed Sensor Networks, vol. 8, no. 8, p. 213980, 2012

[19] S. Zaidi, A. El Assaf, S. Affes, and N. Kandil, "Range-free nodes localization in mobile wireless sensor networks," in 2015 IEEE international conference on ubiquitous wireless broadband (ICUWB). IEEE, 2015, pp. 1-6.

[20] G. Sharma and A. Kumar, "Improved dv-hop localization algorithm using teaching learning based optimization for wireless sensor networks," Telecommunication Systems, vol. 67 , no. 2 , pp. $163-178,2018$.

[21] A. Kaur, P. Kumar, and G. P. Gupta, "Nature inspired algorithm-based improved variants of dv-hop algorithm for randomly deployed 2d and 3d wireless sensor networks," Wireless Personal Communications, vol. 101, no. 1, pp. 567-582, 2018.

[22] S. P. Singh and S. C. Sharma, "Implementation of a pso based improved localization algorithm for wireless sensor networks," IETE Journal of Research, vol. 65, no. 4, pp. 502-514, 2019.

[23] S. Kumar and D. Lobiyal, "An advanced dv-hop localization algorithm for wireless sensor networks," Wireless personal communications, vol. 71 , no. 2, pp. 1365-1385, 2013.

[24] V. Kanwar and A. Kumar, "Multiobjective optimization-based dv-hop localization using nsga-ii algorithm for wireless sensor networks," International Journal of Communication Systems, vol. 33, no. 11, p. e4431, 2020.

[25] R. A. Hinde and J. Fisher, "Further observations on the opening of milk bottles by birds," British Birds, vol. 44, no. 12, pp. 393-396, 1951. 
[26] C. M. Heyes, "Social learning in animals: categories and mechanisms," Biological Reviews, vol. 69, no. 2, pp. 207-231, 1994.

[27] C. Heyes, E. Ray, C. Mitchell, and T. Nokes, "Stimulus enhancement: controls for social facilitation and local enhancement," Learning and Motivation, vol. 31, no. 2, pp. 83-98, 2000.

[28] S. Mineka and M. Cook, "Social learning and the acquisition of snake fear in monkeys," in Social learning. Psychology Press, 2013, pp. 63-86.

[29] H. P. Young, "Innovation diffusion in heterogeneous populations: Contagion, social influence, and social learning," American economic review, vol. 99, no. 5, pp. 18991924,2009

[30] C. F. Bond and L. J. Titus, "Social facilitation: A meta-analysis of 241 studies." Psychological bulletin, vol. 94, no. 2, p. 265, 1983.

[31] T. R. Zentall, "Imitation in animals: evidence, function, and mechanisms," Cybernetics E Systems, vol. 32, no. 1-2, pp. 53-96, 2001.

[32] A. Whiten, "Primate culture and social learning," Cognitive Science, vol. 24, no. 3, pp. 477-508, 2000 .

[33] K. Dautenhahn, C. L. Nehaniv, and A. Alissandrakis, "Learning by experience from others-social learning," Adaptivity and Learning: An Interdisciplinary Debate, p. 217, 2013.

[34] R. W. Mitchell, "A comparative-developmental approach to understanding imitation," in Perspectives in ethology. Springer, 1987, pp. 183-215.

[35] P. Das, D. K. Das, and S. Dey, "A new class topper optimization algorithm with an application to data clustering," IEEE Transactions on Emerging Topics in Computing, 2018.

[36] M. Daneshyari and G. G. Yen, "Cultural-based multiobjective particle swarm optimization," IEEE Transactions on Systems, Man, and Cybernetics, Part B (Cybernetics), vol. 41, no. 2, pp. 553-567, 2010.

[37] J. Vesterstrom and R. Thomsen, "A comparative study of differential evolution, particle swarm optimization, and evolutionary algorithms on numerical benchmark problems," in Proceedings of the 2004 congress on evolutionary computation (IEEE Cat. No. 04TH8753), vol. 2. IEEE, 2004, pp. 1980-1987.

[38] D. Xue, "Research of localization algorithm for wireless sensor network based on $\mathrm{dv}$ hop," EURASIP Journal on Wireless Communications and Networking, vol. 2019 , no. 1, pp. 1-8, 2019 